Bounds for Mixed Strategy Equilibria and the Spatial Model of Elections

Jeffrey S. Banks, John Duggan, and Michel Le Breton

Working Paper No. 14

March 1998
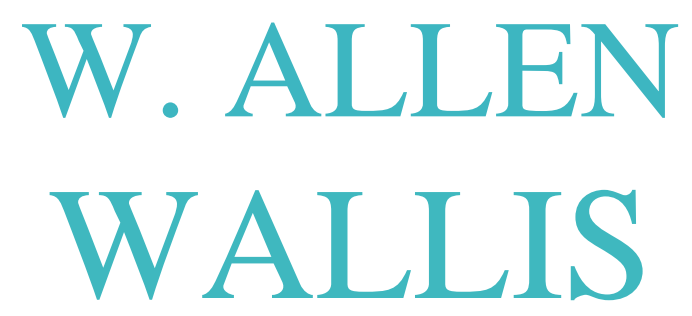

Institute of

POLITICAL

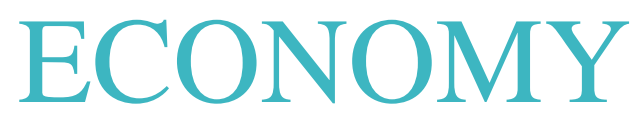

UNIVERSITY OF

ROCHESTER 


\title{
Bounds for Mixed Strategy Equilibria and the Spatial Model of Elections
}

\author{
Jeffrey S. Banks \\ Division of Humanities and Social Sciences \\ California Institute of Technology \\ Pasadena, CA 91125 \\ John Duggan \\ Department of Political Science \\ and Department of Economics \\ University of Rochester \\ Rochester, NY 14627 \\ Michel Le Breton \\ GREQAM-LEQAM \\ Université d'Aix Marseille 2 and \\ Istitut Universitaire de France \\ Route des Milles \\ 13290, Les Milles, France
}

September 14, 1998 


\begin{abstract}
We prove that the support of mixed strategy equilibria of two-player, symmetric, zero-sum games lies in the uncovered set, a concept originating in the theory of tournaments and the spatial theory of politics. We allow for uncountably infinite strategy spaces, and, as a special case, we obtain a longstanding claim to the same effect, due to McKelvey (1986), in the political science literature. Further, we prove the non-emptiness of the uncovered set under quite general assumptions, and we establish, under various assumptions, the measurability and coanalyticity of this set. In the concluding section, we indicate how the inclusion result may be extended to multi-player, non-zero-sum games.
\end{abstract}




\section{Introduction}

A common conclusion drawn from work on the multi-dimensional spatial model of elections is that, typically, anything can happen (Riker (1980)). One strand of work has established that the core, consisting of alternatives unbeaten in pairwise majority comparisons, is generically empty; ${ }^{1}$ another, assuming the core is empty, has deduced the existence of a global cycle. ${ }^{2}$ The situation facing two office-motivated parties might appear, therefore, to be quite indeterminate: from the former strand of work, we know that pure strategy Nash equilibria of the party competition game rarely exist; and from the latter strand, every policy alternative is typically rationalizable. In a seminal paper, McKelvey (1986) claimed to the contrary that the support of mixed strategy equilibria of the game, if they exist, will lie in a centrally located region of the policy space called the "uncovered set." He suggested, moreover, that the uncovered set will collapse, in a continuous way, to the core when it is non-empty; Cox (1987) proved this for an odd number of voters, and Banks, Duggan, and Le Breton (1998) extend the result to a continuum of voters. Thus, the analysis of the uncovered set tells us that equilibrium outcomes will be "close" to the core when the core is "close" to non-empty.

The uncovered set was defined originally by Fishburn (1979) and Miller (1980) and axiomatized by Moulin (1986) in the context of tournaments, i.e., binary relations representing majority preferences of a society over a finite set of policy alternatives. Fisher and Ryan (1991) and Laffond, Laslier, and Le Breton (1993) independently proved that, in the absence of majority-ties, the party competition game has a unique mixed strategy equilibrium and that the support of the agents' strategies lies in the uncovered set. Recently, Dutta and Laslier (1998) have defined the uncovered set in the context of general finite, two-player, symmetric, zero-sum games, and they have shown that the support of each mixed strategy equilibrium (no longer unique) still lies in the uncovered set. ${ }^{3}$

McKelvey's (1986) analysis of the spatial model was the first to consider the uncovered set in an infinite setting, where policy alternatives are

\footnotetext{
${ }^{1}$ Plott (1967), Rubinstein (1979), Schofield (1983), Cox (1984), Le Breton (1987), McKelvey and Schofield (1987), Banks (1995), Saari (1997).

${ }^{2}$ McKelvey (1976, 1979), Cohen (1979), Austen-Smith and Banks (1997).

${ }^{3}$ In fact, more is proved: Laffond, Laslier, and Le Breton (1993) show that the support of the mixed strategy equilibrium lies in the minimal covering set, a smaller set introduced by Dutta (1988) in the context of tournaments; Dutta and Laslier (1998) extend the minimal covering set to finite, two-player, symmetric, zero-sum games and obtain the same inclusion.
} 
modelled as points in a convex subset of Euclidean space, and majority preferences over social policies are determined by the continuous, strictly convex preferences of a finite electorate. Payoffs of the party competition game are, therefore, highly discontinuous, and the existence of mixed strategy equilibria in this model remains an open question. ${ }^{4}$ Nonetheless, McKelvey claims that if a mixed strategy equilibrium does exist, the support of the parties' mixed strategies will necessarily lie in the uncovered set. His proof contains a critical error, ${ }^{5}$ but we confirm that the claim is, indeed, true.

We frame the problem in the more general context of two-player, symmetric, zero-sum games, allowing for infinite strategy spaces. By way of introduction, we first extend the known inclusion results for the uncovered set to the case of games with countable strategy sets, obtaining the result of Dutta and Laslier (1998) as a special case. We then generalize this to the case of games with continuous payoffs and possibly uncountable strategy sets. Because of the discontinuities inherent in the spatial model, however, this level of generality is not sufficient to recover McKelvey's result. We go on to consider games for which the set of strategy profiles yielding a positive payoff is open, a weak continuity condition satisfied in the party competition game studied in McKelvey (1986) and Banks, Duggan, and Le Breton (1998). We prove, as claimed by McKelvey, that the uncovered set includes the support of all mixed strategy equilibria. We also prove that the uncovered set is non-empty, generalizing another result of McKelvey's and a later result of Bordes, Le Breton, and Salles (1992), and we provide an example showing that the inclusion can fail unless some continuity condition is imposed.

Once uncountable strategy sets are allowed for, a number of interesting technical issues arise. Chief among them, it appears that, even when the payoff function of the game is continuous, the uncovered set may be nonmeasurable. To address this difficulty, we employ the theory of analytic sets, developed by Dellacherie and Meyer (1978), surveyed by Stinchcombe and White (1992) and, briefly, in the appendix of this paper. We show that, assuming full continuity, the uncovered set is the complement of an analytic set. Assuming only the weak continuity condition above, it appears that not even this much can be said.

\footnotetext{
${ }^{4}$ Kramer (1978) provides a mixed strategy equilibrium existence result under the assumption of a continuum of voters and vote-maximizing parties.

${ }^{5}$ McKelvey notes that strategies outside the uncovered set are weakly dominated for the parties, and he concludes with the claim that equilibrium strategies cannot put positive probability on "dominated strategies." While this is true of strictly dominated strategies, it is clearly not for weakly dominated ones.
} 


\section{Preliminaries}

Let $X$ be a set of strategies and $u: X \times X \rightarrow \Re$ be a payoff function defining a two-player, zero-sum game, $(X, u)$, symmetric in the sense that $u(x, y)=$ $-u(y, x)$ for all $x, y \in X$. Throughout, $X$ is endowed with a topology (discrete if $X$ is countable) and its Borel $\sigma$-algebra, and $u$ is assumed to be measurable with respect to the product $\sigma$-algebra on $X \times X$. The party competition game is obtained as the special case,

$$
u(x, y)= \begin{cases}1 & \text { if } x P y \\ -1 & \text { if } y P x \\ 0 & \text { else, }\end{cases}
$$

where $P$ is an asymmetric relation on $X$ representing majority preferences. McKelvey (1986) shows, assuming a finite electorate, that continuity of voter preferences is sufficient for $P$ to be open. ${ }^{6}$ We do so elsewhere (Banks, Duggan, and Le Breton (1998)) for an arbitrary electorate. The condition that $P$ is open is, in turn, sufficient for measurability of $u$.

Let $\sigma$ denote a typical mixed strategy, i.e., a Borel probability measure on $X$, and let $\bar{\sigma}$ denote the outer measure induced by $\sigma$; that is,

$$
\bar{\sigma}(Y)=\inf \{\sigma(Z) \mid Z \text { mble, } Y \subseteq Z\}
$$

for all subsets $Y \subseteq X$. When $Y$ is Borel measurable, $\bar{\sigma}(Y)=\sigma(Y)$. The expected payoff (to player 1) from the mixed strategy pair $\left(\sigma, \sigma^{\prime}\right)$ is

$$
\int_{X} \int_{X} u(x, y) \sigma(d x) \sigma^{\prime}(d y)
$$

or, by Fubini's theorem,

$$
\int_{X \times X} u(x, y)\left(\sigma \times \sigma^{\prime}\right)(d(x, y)),
$$

where $\sigma \times \sigma$ is the product measure induced by the mixed strategies. The pair $\left(\sigma, \sigma^{\prime}\right)$ constitutes a Nash equilibrium if, for all mixed strategies $\hat{\sigma}$, the following inequalities hold:

$$
\begin{aligned}
\int_{X} \int_{X} u(x, y) \hat{\sigma}(d x) \sigma^{\prime}(d y) & \leq \int_{X} \int_{X} u(x, y) \sigma(d x) \sigma^{\prime}(d y) \\
\int_{X} \int_{X} u(x, y) \sigma(d x) \hat{\sigma}(d y) & \geq \int_{X} \int_{X} u(x, y) \sigma(d x) \sigma^{\prime}(d y) .
\end{aligned}
$$

\footnotetext{
${ }^{6}$ In fact, McKelvey considers social preferences derived from general simple games. Our results apply equally well in this setting.
} 
It is clear that, if $\left(\sigma, \sigma^{\prime}\right)$ is a Nash equilibrium, neither $\sigma$ nor $\sigma^{\prime}$ can put positive probability on the set of strictly dominated strategy profiles. We pursue a tighter bound on the support of mixed strategy equilibria.

Our analysis employs the following binary relations on $X$.

$$
\begin{array}{ll}
x D y \quad(\forall z \in X, u(x, z) \geq u(y, z)) \&(\exists z \in X, u(x, z)>u(y, z)) \\
x M y \quad(u(x, y)>0) \&(\forall z \in X, u(y, z)>0 \Rightarrow u(x, z)>0) \\
x G y \quad(u(x, y)>0) \&(\forall z \in X, u(z, x)>0 \Rightarrow u(z, y)>0) \\
x C y \quad(u(x, y)>0) \& x D y
\end{array}
$$

Here, $D$ is the usual notion of weak dominance; $M$ is the Miller relation, introduced by Miller (1980) in the context of majority voting; $G$ is the Gillies relation, introduced by Gillies (1959) in his analysis of zero-sum games and proposed by Fishburn (1979) in the context of majority voting; and $C$ is the covering relation, defined by McKelvey (1986) in the context of the spatial voting model. Note that $M$ and $G$ are generally stronger than $C$, and that the relations are equivalent in the majority voting model without ties (i.e., $x \neq y$ implies $u(x, y) \in\{1,-1\})$. Each of the relations defined above is a strict partial order and, if $X$ is finite, it follows that each possesses maximal elements. In Section 4, we give general conditions sufficient for the existence of $G$-maximal, and therefore $C$-maximal, strategies.

The rationalizable strategies of Bernheim (1984) and Pearce (1984) give bounds on the support of mixed strategy equilibria. As the next examples show, there is no general inclusion relation between this set and the maximal strategies of the above relations. Payoffs are given for the row player.

Example 1. Here, $x C z$ but $z$ is rationalizable as a best response to $y$, which is a best response to $w$, which is a best response to $z$.

[Figure 1 here.]

Example 2. Here, $x$ is $C$-maximal but strictly dominated for the row player by the mixed strategy $(0,1 / 2,1 / 2)$, and therefore it is not rationalizable.

[Figure 2 here.]

See Duggan and Le Breton (1998) for an example of the majority voting model in which a $C$-maximal strategy is not rationalizable. Thus, the conclusion of Example 2 carries over to this important special case of games. 
Duggan and Le Breton show, however, that, in the absence of ties, the $C$-maximal strategies are always rationalizable.

As in Examples 3 and 4, below, weakly dominated strategies may be played with positive probability in equilibrium. Moreover, they show that the sets of $M$ - and $G$-maximal elements are too small to bound the support of mixed strategy equilibria, even in the majority voting model.

Example 3. Let $X=\{x, y, z\}$ with payoffs below. Note that $((0,1 / 2,1 / 2)$, $(0,1 / 2,1 / 2))$ is a Nash equilibrium, but $x M y$.

[Figure 3 here.]

Example 4. Let $X=\{x, y, z, w\}$ with payoffs below. Note that $((0,1 / 3,1 / 3$, $1 / 3),(0,1 / 3,1 / 3,1 / 3))$ is a Nash equilibrium, but $x G y$.

[Figure 4 here.]

Thus, of the relations introduced above, we focus on the maximal elements of the covering relation for a bound on the support of mixed strategy equilibria. The uncovered set, denoted $U$, consists of the maximal elements of the covering relation: $x \in U$ if and only if there is no $y \in X$ such that $y C x$. Letting $V=X \backslash U$ denote the set of covered strategies, we can view $C: V \rightarrow \longrightarrow X$ as a correspondence, with graph $C=\{(x, y) \in X \times X \mid y \in$ $C(x)\}$.

In the appendix, we prove a lemma on the measurable structure of graph $C$ and its projection, $V$. Under the most general assumptions, we can say little; adding continuity of $u$, graph $C$ is measurable and $V$ is analytic; adding compactness of $X$, both sets (and therefore $U$ ) are measurable.

\section{Continuous Games}

The uncovered set has received much attention in the context of tournaments, including an axiomatic characterization by Moulin (1986); Laffond, Laslier, and Le Breton (1993) have proved, as a corollary of their result on the minimal covering set, that the support of the unique mixed strategy equilibrium is included in the uncovered set; and Dutta and Laslier (1998) have extended this to finite, two-player, symmetric, zero-sum games. Our first result, mainly illustrative, verifies the inclusion for countable, two-player, symmetric, zero-sum games. 
Theorem 1. If $X$ is countable and $\left(\sigma, \sigma^{\prime}\right)$ is a Nash equilibrium then $\sigma(U)=\sigma^{\prime}(U)=1$.

Proof. Suppose that $\sigma(U)<1$ and note that, by interchangeability, $(\sigma, \sigma)$ is a Nash equilibrium. Since $V$ is countable and $\sigma(V)>0$, there exists $x \in V$ and $y \in C(x)$ such that $\sigma(\{x\})>0$. Now consider the mixed strategy $\hat{\sigma}$ defined by $\hat{\sigma}(\{y\})=1$. Since $u(y, x)>0=u(x, x)$, we have

$$
\begin{aligned}
& \int_{X} \int_{X} u\left(x^{\prime}, x^{\prime \prime}\right) \hat{\sigma}\left(d x^{\prime}\right) \sigma\left(d x^{\prime \prime}\right) \\
& =\int_{X} u\left(y, x^{\prime \prime}\right) \sigma\left(d x^{\prime \prime}\right) \\
& =\sigma(\{x\}) u(y, x)+\int_{X \backslash\{x\}} u\left(y, x^{\prime \prime}\right) \sigma\left(d x^{\prime \prime}\right) \\
& >\sigma(\{x\}) u(x, x)+\int_{X \backslash\{x\}} u\left(x, x^{\prime \prime}\right) \sigma\left(d x^{\prime \prime}\right) \\
& =\int_{X} \int_{X} u\left(x^{\prime}, x^{\prime \prime}\right) \sigma\left(d x^{\prime}\right) \sigma\left(d x^{\prime \prime}\right),
\end{aligned}
$$

contradicting the fact that $(\sigma, \sigma)$ is a Nash equilibrium. A symmetric argument addresses the case $\sigma^{\prime}(U)<1$ and completes the proof.

We now extend the arguments in support of Theorem 1 to show that, assuming payoff functions are continuous and $X$ is second countable (i.e., the topology on $X$ has a countable base), the supports of Nash equilibria are included within the uncovered set. This increased generality raises two technical challenges: first, the set $V$ is analytic but, it appears, may be non-measurable; second, there may no longer be a strategy $x \in V$ such that $\sigma(\{x\})>0$.

Theorem 2. If $X$ is second countable, $u$ is continuous, and $\left(\sigma, \sigma^{\prime}\right)$ is a Nash equilibrium, then $\bar{\sigma}(U)=\bar{\sigma}^{\prime}(U)=1$. If, in addition, $X$ is a complete, separable metric space, then there exists a measurable set $\hat{U} \subseteq U$ such that $\sigma(\hat{U})=\sigma^{\prime}(\hat{U})=1$.

Proof. Suppose that $\bar{\sigma}(U)<1$, so there exists a measurable set $U^{\prime} \supseteq U$ such that $\sigma\left(U^{\prime}\right)<1$. Letting $V^{\prime}=X \backslash U^{\prime}$, we have $V^{\prime} \subseteq V$ and $\sigma\left(V^{\prime}\right)>0$. Note that, by interchangeability, $(\sigma, \sigma)$ is a Nash equilibrium. Let $\hat{V}$ be the measurable set consisting of strategies $x \in V^{\prime}$ such that

$$
\int_{X} u\left(x, x^{\prime \prime}\right) \sigma\left(d x^{\prime \prime}\right) \geq \int_{X} \int_{X} u\left(x^{\prime}, x^{\prime \prime}\right) \sigma\left(d x^{\prime}\right) \sigma\left(d x^{\prime \prime}\right),
$$

and note that, because $(\sigma, \sigma)$ is a Nash equilibrium, $\sigma(\hat{V})=\sigma\left(V^{\prime}\right)$. For 
all $x \in \hat{V}$ and all $y \in C(x), u(y, x)>0=u(x, x)$. Thus, continuity of $u$ yields an open set $Z_{x, y} \subseteq X$ such that $x \in Z_{x, y}$ and, for all $z \in Z_{x, y}$, $u(y, z)>u(x, z)$.

Now consider $\hat{V}$ as a topological space endowed with the relative topology induced by $X$. This space is second countable and, therefore, Lindelöf: every open cover contains a countable subcover. Since $\left\{Z_{x, y} \cap \hat{V} \mid x \in \hat{V}, y \in C(x)\right\}$ is an open cover of $V^{\prime}$, it has a countable subcover $\left\{Z_{x_{k}, y_{k}} \cap \hat{V} \mid k=1,2, \ldots\right\}$.

Since $\sigma\left(\bigcup_{k=1}^{\infty} Z_{x_{k}, y_{k}}\right) \geq \sigma(\hat{V})>0$, there exists a $k$ such that $\sigma\left(Z_{x_{k}, y_{k}}\right)>$ 0 . Then the expected payoff from $(\hat{\sigma}, \sigma)$, where $\hat{\sigma}$ is defined by $\hat{\sigma}\left(\left\{y_{k}\right\}\right)=1$, is

$$
\begin{aligned}
& \int_{Z_{x_{k}, y_{k}}} u\left(y_{k}, x^{\prime \prime}\right) \sigma\left(d x^{\prime \prime}\right)+\int_{X \backslash Z_{x_{k}, y_{k}}} u\left(y_{k}, x^{\prime \prime}\right) \sigma\left(d x^{\prime \prime}\right) \\
& \quad \geq \int_{Z_{x_{k}, y_{k}}} u\left(y_{k}, x^{\prime \prime}\right) \sigma\left(d x^{\prime \prime}\right)+\int_{X \backslash Z_{x_{k}, y_{k}}} u\left(x_{k}, x^{\prime \prime}\right) \sigma\left(d x^{\prime \prime}\right) \\
& =\int_{Z_{x_{k}, y_{k}}}\left[u\left(y_{k}, x^{\prime \prime}\right)-u\left(x_{k}, x^{\prime \prime}\right)\right] \sigma\left(d x^{\prime \prime}\right)+\int_{X} u\left(x_{k}, x^{\prime \prime}\right) \sigma\left(d x^{\prime \prime}\right) \\
& >\int_{X} u\left(x_{k}, x^{\prime \prime}\right) \sigma\left(d x^{\prime \prime}\right) \\
& \geq \int_{X} \int_{X} u\left(x^{\prime}, x^{\prime \prime}\right) \sigma\left(d x^{\prime}\right) \sigma\left(d x^{\prime \prime}\right),
\end{aligned}
$$

where the first inequality follows from $y_{k} D x_{k}$; the second from $u\left(y_{k}, z\right)>$ $u\left(x_{k}, z\right)$ for all $z \in Z_{x_{k}, y_{k}}$ and $\sigma\left(Z_{x_{k}, y_{k}}\right)>0$; and the third from condition (1). This contradicts the presumption that $(\sigma, \sigma)$ is a Nash equilibrium. A symmetric argument addresses the case $\bar{\sigma}^{\prime}(U)<1$.

If $X$ is a complete, separable metric space, then, by Lemma $1, V$ is analytic. Thus, there are measurable sets $Y \supseteq V$ and $Z \supseteq V$ such that $\sigma(Y)=\sigma^{\prime}(Z)=0$. Setting $\hat{U}=X \backslash(Y \cap Z)$, the proof is complete.

By Lemma 1, in the appendix, if $X$ is a compact metric space, $U$ is itself measurable and we can take $\hat{U}=U$ in the statement of Theorem 2 .

\section{Discontinuous Games}

Adding compactness of $X$ to the assumptions of Theorem 2, Nash equilibria exist (see Glicksberg (1952)), and, as a consequence, the uncovered set is non-empty. The next theorem establishes that $G$-maximal, and therefore uncovered, strategies actually exist under very weak continuity assumptions. This result generalizes McKelvey's (1986) existence theorem, which assumes 
strict convexity of voter preferences, and it generalizes the result of Bordes, Le Breton, and Salles (1992) by dropping the metric space structure of $X$ and weakening continuity.

Theorem 3. If $X$ is compact and $\{y \in X \mid u(x, y)>0\}$ is open for all $x \in X$, then $G$-maximal elements exist.

Proof. We first define the following (transitive) relations.

$$
\begin{aligned}
& x G^{\circ} y \quad \forall z \in X, u(z, x)>0 \Rightarrow u(z, y)>0 \\
& x G^{*} y \quad x G^{\circ} y \& \neg y G^{\circ} x
\end{aligned}
$$

Every $G^{*}$-maximal element is a $G$-maximal element, so it suffices to prove the existence of the former. To this end, let $\left\{x_{\alpha}\right\}$ be a $G^{*}$-chain in $X$. We will show that this chain has a $G^{*}$-upper bound in $X$, so that Zorn's lemma yields a $G^{*}$-maximal element.

If $\left\{x_{\alpha}\right\}$ contains a $G^{*}$-upper bound, we are done. Therefore, suppose otherwise. By compactness, $\left\{x_{\alpha}\right\}$ has an accumulation point, say $x$. We first show that $x$ is a $G^{\circ}$-upper bound of $\left\{x_{\alpha}\right\}$ by taking arbitrary $x_{\alpha}$ and $z \in X$ such that $u(z, x)>0$. By assumption, there exists an open set $Y$ such that $x \in Y$ and, for all $y \in Y, u(z, y)>0$. Because $x$ is an accumulation point, there exists $\alpha^{\prime}>\alpha$ such that $x_{\alpha^{\prime}} \in Y$, implying $u\left(z, x_{\alpha^{\prime}}\right)>0$. Then $x_{\alpha^{\prime}} G^{*} x_{\alpha}$ implies $u\left(z, x_{\alpha}\right)>0$, from which we conclude that $x G^{\circ} x_{\alpha}$.

We now show that $x$ is a $G^{*}$-upper bound, again taking arbitrary $x_{\alpha}$. If $x_{\alpha} G^{\circ} x$ then, by transitivity of $G^{\circ}$ and the fact that $x$ is a $G^{\circ}$-upper bound of $\left\{x_{\alpha}\right\}$, it follows that $x_{\alpha}$ is a $G^{\circ}$-upper bound. Because $\left\{x_{\alpha}\right\}$ is a $G^{*}$-chain, this means that $x_{\alpha}$ is, in fact, a $G^{*}$-upper bound, contrary to our initial supposition. Thus, the proof is complete.

Our final theorem generalizes the second part of Theorem 2 by substantially weakening continuity. The weaker assumption is clearly satisfied in the majority voting model when $P$ is open, delivering McKelvey's (1986) stated inclusion result as a special case. The possibility of discontinuous payoffs adds to the technical challenges of Theorem 2: because we cannot conclude, under the assumptions of the theorem, that graph $C$ is measurable, we do not know that $V$ is even analytic. In the proof, we are forced to approximate graph $C$ from above by an appropriately chosen measurable set. ${ }^{7}$

\footnotetext{
${ }^{7} \mathrm{~A}$ more subtle point is that, because $u$ is not continuous, we cannot find sets $Z_{x, y}$ as in the proof of Theorem 2, and we cannot directly find a pure strategy deviation with positive expected payoff. We are forced to use a selection from the measurable approximation of graph $C$.
} 
Theorem 4. If $X$ is a complete, separable metric space, $\{(x, y) \in X \times X \mid$ $u(x, y)>0\}$ is open, and $\left(\sigma, \sigma^{\prime}\right)$ is a Nash equilibrium, then there exists a measurable set $\hat{U} \subseteq U$ such that $\sigma(\hat{U})=\sigma^{\prime}(\hat{U})=1$.

Proof. By interchangeability, $(\sigma, \sigma)$ and $\left(\sigma^{\prime}, \sigma^{\prime}\right)$ are both Nash equilibria, so we focus on $\sigma$ without loss of generality. Define the following two relations on $X$.

$$
\begin{array}{ll}
x D^{\epsilon} y & \sigma(\{w \in X \mid u(y, w)>u(x, w)\})<\epsilon \\
x C^{\epsilon} y & x D^{\epsilon} y \& u(x, y)>0 \\
x C^{*} y \quad \forall \epsilon>0, x C^{\epsilon} y
\end{array}
$$

Thus, $x D^{\epsilon} y$ if the set of strategies against which $y$ gives a higher payoff than $x$ has measure less than $\epsilon ; x C^{\epsilon} y$ adds $u(x, y)>0$; and $x C^{*} y$ if $u(x, y)>0$ and the set of strategies against which $y$ gives a higher payoff than $x$ has measure zero. Clearly, $x C y$ implies $x C^{*} y$, or, alternatively,

$$
\operatorname{graph} C \subseteq \operatorname{graph} C^{*}=\bigcap_{k=1}^{\infty} \operatorname{graph} C^{1 / k} .
$$

Define $V^{\prime}=\operatorname{proj}_{x}$ graph $C^{*}$, and note that $V \subseteq V^{\prime}$.

We claim that graph $C^{\epsilon}$, and therefore graph $C^{*}$, is measurable. Writing $\sigma(\{w \in X \mid u(y, w)>u(x, w)\})$ as

$$
\int_{X} \chi_{\{w \mid u(y, w)>u(x, w)\}}(z) \sigma(d z)
$$

this will follow if $\chi_{\{w \mid u(y, w)>u(x, w)\}}(z)$ is a measurable function of $x, y$, and $z$. Note that $(x, y, z)$ satisfies $\chi_{\{w \mid u(y, w)>u(x, w)\}}(z)=1$ if and only if $u(y, z)>$ $u(x, z)$, so, because $u$ is measurable, these triples comprise a measurable set, finishing the claim. As a consequence, $V^{\prime}$ is analytic.

Suppose there exists a measurable subset $\hat{V} \subseteq V^{\prime}$ such that $\sigma(\hat{V})>0$. Since $\left(\right.$ graph $\left.C^{*}\right) \cap(\hat{V} \times X)$ is measurable, Dellacherie and Meyer's (1978) Theorem III.44 (and remarks following) yields a measurable "almost everywhere selection" of $\left(\right.$ graph $\left.C^{*}\right) \cap(\hat{V} \times X)$. That is, there is a measurable function $\gamma: X \rightarrow X$ such that, for $\sigma$-almost every $x \in \hat{V},(x, \gamma(x)) \in$ $\left(\operatorname{graph} C^{*}\right) \cap(\hat{V} \times X)$. Applying Lusin's theorem (see Rudin (1966)), there is a measurable subset $\tilde{V} \subseteq \hat{V}$ such that $\sigma(\tilde{V})>0$ and $\gamma$ is continuous on $\tilde{V}$. Define $\tilde{\gamma}: X \rightarrow X$ by

$$
\tilde{\gamma}(x)= \begin{cases}\gamma(x) & \text { if } x \in \tilde{V} \\ x & \text { else }\end{cases}
$$


also a measurable function.

Now consider the deviation $\tilde{\sigma}=\sigma \circ \tilde{\gamma}$, meaning $\tilde{\sigma}(Y)=\sigma\left(\tilde{\gamma}^{-1}(Y)\right)$ for each measurable $Y$. For all $x \in \tilde{V}, u(\tilde{\gamma}(x), x)>0$, so, using the weak continuity assumption of the theorem, there exists $\delta_{x}>0$ such that

$$
\forall w \in B_{\delta_{x}}(x), \forall y \in B_{\delta_{x}}(\tilde{\gamma}(x)), u(y, w)>0 .
$$

Because $\tilde{\gamma}$ is continuous on $\tilde{V}, \tilde{\gamma}^{-1}\left(B_{\delta_{x}}(\tilde{\gamma}(x))\right)$ includes a relatively open set around $x$. Choose $\epsilon_{x} \in\left(0, \delta_{x}\right)$ such that

$$
B_{\epsilon_{x}}(x) \cap \tilde{V} \subseteq \tilde{\gamma}^{-1}\left(B_{\delta_{x}}(\tilde{\gamma}(x))\right) .
$$

Since $(x, \tilde{\gamma}(x)) \in B_{\epsilon_{x}}(x) \times B_{\epsilon_{x}}(\tilde{\gamma}(x))$ for all $x \in \tilde{V}$, it follows that $\left\{B_{\epsilon_{x}}(x) \times\right.$ $\left.B_{\epsilon_{x}}(\tilde{\gamma}(x)) \mid x \in \tilde{V}\right\}$ is an open cover of $\{(x, \tilde{\gamma}(x)) \mid x \in \tilde{V}\}$. By the Lindelöf property, it has a countable subcover, $\left\{B_{k}\left(x_{k}\right) \times B_{k}\left(\tilde{\gamma}\left(x_{k}\right)\right) \mid k=1,2, \ldots\right\}$, where we write $B_{k}\left(x_{k}\right)$ for $B_{\epsilon_{x_{k}}}\left(x_{k}\right)$.

Since $\left\{B_{k}\left(x_{k}\right) \cap \tilde{V} \mid k=1,2, \ldots\right\}$ covers $\tilde{V}$, we have

$$
\sigma\left(\bigcup_{k=1}^{\infty} B_{k}\left(x_{k}\right) \cap \tilde{V}\right)=\sigma(\tilde{V})>0 .
$$

In particular, $\sigma\left(B_{k}\left(x_{k}\right) \cap \tilde{V}\right)>0$ for some $k$. The expected payoff from $(\tilde{\sigma}, \sigma)$ is then

$$
\begin{aligned}
\int_{X} & \int_{X} u\left(x^{\prime}, x^{\prime \prime}\right) \tilde{\sigma}\left(d x^{\prime}\right) \sigma\left(d x^{\prime \prime}\right) \\
= & \int_{B_{k}\left(x_{k}\right) \times B_{k}\left(x_{k}\right)} u\left(\tilde{\gamma}\left(x^{\prime}\right), x^{\prime \prime}\right)(\sigma \times \sigma)\left(d\left(x^{\prime}, x^{\prime \prime}\right)\right) \\
& +\int_{(X \times X) \backslash\left(B_{k}\left(x_{k}\right) \times B_{k}\left(x_{k}\right)\right)} u\left(\tilde{\gamma}\left(x^{\prime}\right), x^{\prime \prime}\right)(\sigma \times \sigma)\left(d\left(x^{\prime}, x^{\prime \prime}\right)\right) \\
> & \int_{B_{k}\left(x_{k}\right) \times B_{k}\left(x_{k}\right)} u\left(x^{\prime}, x^{\prime \prime}\right)(\sigma \times \sigma)\left(d\left(x^{\prime}, x^{\prime \prime}\right)\right) \\
& +\int_{(X \times X) \backslash\left(B_{k}\left(x_{k}\right) \times B_{k}\left(x_{k}\right)\right)} u\left(x^{\prime}, x^{\prime \prime}\right)(\sigma \times \sigma)\left(d\left(x^{\prime}, x^{\prime \prime}\right)\right) \\
= & \int_{X} \int_{X} u\left(x^{\prime}, x^{\prime \prime}\right) \sigma\left(d x^{\prime}\right) \sigma\left(d x^{\prime \prime}\right) .
\end{aligned}
$$

The above equality follows from the change of variables theorem, and the inequality follows from three observations. First, by symmetry and the assumption that $(X, u)$ is zero-sum,

$$
\int_{B_{k}\left(x_{k}\right) \times B_{k}\left(x_{k}\right)} u\left(x^{\prime}, x^{\prime \prime}\right)(\sigma \times \sigma)\left(d\left(x^{\prime}, x^{\prime \prime}\right)\right)=0 .
$$


Second, from (3), we have $\tilde{\gamma}(w) \in B_{\delta_{x_{k}}}\left(\tilde{\gamma}\left(x_{k}\right)\right)$ for all $w \in B_{k}\left(x_{k}\right)$; and from (2), this implies that $u(\tilde{\gamma}(w), z)>0$ for all $w, z \in B_{k}\left(x_{k}\right)$; and then $\sigma\left(B_{k}\left(x_{k}\right)\right)>0$ yields

$$
\int_{B_{k}\left(x_{k}\right) \times B_{k}\left(x_{k}\right)} u\left(\tilde{\gamma}\left(x^{\prime}\right), x^{\prime \prime}\right)(\sigma \times \sigma)\left(d\left(x^{\prime}, x^{\prime \prime}\right)\right)>0 .
$$

Third, for all $x \in \tilde{V}$ and $\sigma$-almost every $w, u(\tilde{\gamma}(x), w) \geq u(x, w)$. Thus, we have contradicted the presumption that $(\sigma, \sigma)$ is a Nash equilibrium.

Therefore, there is no measurable subset of $V^{\prime}$ with positive measure. Because $V^{\prime}$ is analytic, there there are measurable sets $Y \subseteq V^{\prime}$ and $Z \supseteq V^{\prime}$ such that $\sigma(Y)=\sigma^{\prime}(Z)$. We have just shown that $\sigma(Y)=0$. Setting $\hat{U}=X \backslash Z$ and noting that $V \subseteq V^{\prime}$, the proof is complete.

Our last example demonstrates that Theorem 4 cannot be stated without some continuity condition on $u$.

Example 5. Let $X=[0,1]$ with payoffs below, where the solid diagonal line contains entries equal to 1 ; the dashed diagonal line contains entries equal to -1 ; and all other entries are 0 . Here, every strategy $x \in[0,1 / 2)$ is covered by $x+1 / 2$, but the strategies defined by the uniform probability distribution on $[0,1 / 2)$ constitute a Nash equilibrium.

[Figure 5 here.]

\section{$5 \quad$ Extensions}

Though defined in the context of two-player, symmetric, zero-sum games, the covering relation is easily extended to general symmetric games. Let $\left(X, u_{1}, u_{2}\right)$ denote a (possibly non-zero-sum) game, symmetric in the sense that $u_{1}(x, y)=u_{2}(y, x)$ for all $x, y \in X$. We modify the definition of covering as follows.

$$
x C y \quad u_{1}(x, y)>u_{1}(y, y) \& x D y
$$

As with the zero-sum case, the uncovered set is a superset of the undominated strategies. In the Bertrand model of competition between two pricesetting firms, for example, the unique Nash equilibrium is for both firms to announce price equal to marginal cost - a strategy weakly dominated for both firms yet uncovered, suggesting that the inclusion results of Section 4 may be more general than stated there.

We now generalize the proof of Theorem 2 in a sequence of steps (for simplicity, considering only the first part of the theorem), relying on the original proof for our arguments: 
Assume $X$ is second countable, $\left(X, u_{1}, u_{2}\right)$ is symmetric with continuous payoffs and interchangeable equilibria. If $\left(\sigma, \sigma^{\prime}\right)$ is a Nash equilibrium, then $\bar{\sigma}(U)=\bar{\sigma}^{\prime}(U)=1$.

We can easily drop the assumption of interchangeability by restating the result for symmetric equilibria:

Assume $X$ is second countable and $\left(X, u_{1}, u_{2}\right)$ is symmetric with continuous payoffs. If $(\sigma, \sigma)$ is a Nash equilibrium, then $\bar{\sigma}(U)=$ 1 .

Furthermore, we can relax the symmetry requirement, defining covering as above but with the interpretation of "covering for player 1." Letting $U^{1}$ denote the set of strategies uncovered for player 1, the following extension is straightforward:

If $X$ is second countable, $u_{1}$ is continuous, and $(\sigma, \sigma)$ is a Nash equilibrium, then $\bar{\sigma}\left(U^{1}\right)=1$.

Define a strategy pair $\left(\sigma, \sigma^{\prime}\right)$ to be mutually absolutely continuous if, for all measurable $Y \subseteq X, \sigma(Y)=0$ if and only if $\sigma^{\prime}(Y)=0$. This lets us generalize the result to equilibria that are, in at least a limited sense, non-symmetric:

If $X$ is second countable, $u_{1}$ is continuous, and $\left(\sigma, \sigma^{\prime}\right)$ is a mutually absolutely continuous Nash equilibrium, then $\bar{\sigma}\left(U^{1}\right)=1$.

Finally, for an $n$-player game $\left(X, u_{1}, \ldots, u_{n}\right)$, define $x$ to cover $y$ for player $i$ if $u_{i}(x, y, \ldots, y)>u_{i}(y, \ldots, y)$ and $x D y$. The reader can check that the proof of Theorem 2 is easily modified to support our last generalization of the theorem:

If $X$ is second countable, $u_{1}$ is continuous, and $\left(\sigma_{1}, \ldots, \sigma_{n}\right)$ is a mutually absolutely continuous Nash equilibrium, then $\bar{\sigma}_{1}\left(U^{1}\right)=$ 1.

Unfortunately, Theorem 4 is not so easily generalized, because equality (4) relies on the symmetric, zero-sum structure of the game. The example of the Bertrand model suggests that it does generalize, but we leave this question open.

\section{A Appendix}

Let $(W,-)$ be a measurable space. A set $Y \subseteq W$ is analytic if there exists a compact metric space, $E$, with its Borel $\sigma$-algebra, and a measurable 
set $B \subseteq W \times E$, endowed with the product $\sigma$-algebra, such that $Y$ is the projection of $B$. Every measurable subset of $W$ is analytic, but there are some analytic sets that are not measurable. A set $Y$ is coanalytic if it is the complement of an analytic set. Analyticity is preserved by countable intersections and unions, but not complementation. Thus, coanalytic sets may not be analytic.

The analytic sets are close to measurable, however. If $Y$ is analytic then, for any probability measure $\mu$ on $(W,-)$, there are measurable sets $S$ and $R$ such that $S \subseteq Y \subseteq R$ and $\mu(S)=\mu(R)$.

A function $f: X \rightarrow \Re$ is analytic if, for all $c,\{x \in X \mid f(x)>r\}$ is analytic; equivalently, if, for all $c,\{x \in X \mid f(x) \geq r\}$ is analytic.

An important virtue of analytic sets is the following. Let $S$ be a complete, separable metric space (and, therefore, a Souslin space), and let $A$ be an analytic subset of $S \times W$. Then the projection of $A$ onto $W$ is analytic. In contrast, we can say nothing about the projection of coanalytic sets.

See Dellacherie and Meyer (1978) for a complete treatment of analytic sets and functions, or see Stinchcombe and White (1992) for a good survey.

\section{Lemma 1.}

1. If $X$ is a complete, separable metric space, then graph $C$ is coanalytic.

2. If $u$ is continuous, then graph $C$ is measurable. If, in addition, $X$ is a complete, separable metric space, then $V$ is analytic.

3. If $X$ is a compact metric space and $u$ is continuous, then $V$ (and therefore $U$ ) is measurable.

Proof. 1. Define $f: X \times X \times X \rightarrow \bar{\Re}$ by $f(x, y, z)=u(x, z)-u(y, z)$. Because $u$ is measurable, $f$ is measurable with respect to the product $\sigma$-algebra on $X \times X \times X$. Define $f^{*}: X \times X \rightarrow \bar{\Re}$ by $f^{*}(x, y)=\sup _{z \in X} f(x, y, z)$. Вy Stinchcombe and White's (1992) Theorem 2.17, $f^{*}$ is analytic, and it follows that $\left\{(x, y) \in X \times X \mid f^{*}(x, y)>0\right\}$ is an analytic set. Noting that graph $C$ is the complement of

$$
\{(x, y) \in X \times X \mid u(x, y) \geq 0\} \cup\left\{(x, y) \in X \times X \mid f^{*}(x, y)>0\right\},
$$

an analytic set, we see that graph $C$ is coanalytic.

2. If $u$ is continuous, then $f$ is also. Note that $\{(x, y) \in X \times X \mid$ $\left.f^{*}(x, y)>0\right\}$ is the projection of the open set

$$
\{(x, y, z) \in X \times X \times X \mid f(x, y, z)>0\},
$$


and is therefore itself open. Then graph $C$ is the complement of a measurable set, as above, and, therefore, measurable. The set $V$ is the projection of $\operatorname{graph} C$, and, if $X$ is a complete, separable metric space, $V$ is therefore analytic.

3. Now add the assumption that $X$ is compact. By the theorem of the maximum, $f^{*}$ is continuous, implying that $\left\{(x, y) \in X \times X \mid f^{*}(x, y) \geq 0\right\}$ is closed. Then, from the above analysis, each section $C(x)$ is the intersection of an open set and a closed set, and thus $C(x)$ can be written as the countable union of compact subsets of $X$. Using Stinchcombe and White's (1992) Fact 2.2 , as in the proof of their Example 2.12, it follows that $V=\operatorname{proj}_{x}$ graph $C$ is measurable. (See also Larman (1973), Corollary to Theorem 1.) Then, of course, $U=X \backslash V$ is measurable as well.

\section{References}

[1] D. Austen-Smith and J. Banks (1997) "Cycling of Simple Rules in the Spatial Model," mimeo.

[2] J. Banks (1995) "Singularity Theory and Core Existence in the Spatial Model," Journal of Mathematical Economics, 24: 523-536.

[3] J. Banks, J. Duggan, and M. Le Breton (1998) "The Spatial Model of Elections with Arbitrary Distributions of Voters," mimeo.

[4] D. Bernheim (1984) "Rationalizable Strategic Behavior," Econometrica, 52: 1007-1028.

[5] G. Bordes, M. Le Breton, and M. Salles (1992) "Gillies and Miller's Subrelations of a Relation over an Infinite Set of Alternatives: General Results and Applications to Voting Games," Mathematics of Operations Research, 17: 509-518.

[6] L. Cohen (1979) "Cycle Sets in Multidimensional Voting Models," Journal of Economic Theory, 20: 1-12.

[7] G. Cox (1984) "Non-collegial Simple Games and the Nowhere Denseness of the Set of Preference Profiles Having a Core," Social Choice and Welfare, 1: 159-164.

[8] G. Cox (1987) "The Uncovered Set and the Core," American Journal of Political Science, 31: 408-422. 
[9] C. Dellacherie and P.-A. Meyer (1978) Probabilities and Potential, New York: North-Holland.

[10] J. Duggan and M. Le Breton (1998) "Mixed Refinements of Shapley's Saddles and Weak Tournaments," mimeo.

[11] B. Dutta (1988) "Covering Sets and a New Condorcet Choice Correspondence," Journal of Economic Theory, 44: 63-80.

[12] D. Fisher and J. Ryan (1991) "Optimal Strategies for a Generalized 'Scissors, Paper, and Stone' Game," mimeo.

[13] P. Fishburn (1977) "Condorcet Social Choice Functions," SIAM Journal of Applied Mathematics, 33: 469-489.

[14] D. Gillies (1959) "Solutions to General Non-zero-sum Games," in Contributions to the Theory of Games IV, A. Tucker and R. Luce, ed., Annals of Mathematics Studies, no. 40, Princeton: Princeton University Press.

[15] I. Glicksberg (1952) "A Further Generalization of the Kakutani Fixed Point Theorem, with Applications to Nash Equilibrium Points," Proceedings ofthe American Mathematical Society, 3: 170-174.

[16] G. Kramer (1978) "Existence of Electoral Equilibrium," in Game Theory and Political Science, P. Ordeshook, ed., New York: New York University Press.

[17] G. Laffond, J. Laslier, and M. Le Breton (1993) "The Bipartisan Set of a Tournament Game," Games and Economic Behavior, 5: 182-201.

[18] D. Larman (1973) "Projecting and Uniformising Borel Sets with $K_{\sigma^{-}}$ Sections II," Mathematika, 20: 233-246.

[19] M. Le Breton (1987) "On the Core of Voting Games," Social Choice and Welfare, 4: 295-305.

[20] R. McKelvey (1976) "Intransitivities in Multi-dimensional Voting Models and Some Implications for Agenda Control," Journal of Economic Theory, 12: 472-482.

[21] R. McKelvey (1979) "General Conditions for Global Intransitivities in Formal Voting Models," Econometrica, 47: 1086-1112. 
[22] R. McKelvey (1986) "Covering, Dominance, and Institution-free Properties of Social Choice," American Journal of Political Science, 30: 283314 .

[23] R. McKelvey and N. Schofield (1987) "Generalized Symmetry Conditions at a Core Point," Econometrica, 55: 923-934.

[24] N. Miller (1980) "A New Solution Set for Tournaments and Majority Voting: Further Graph-theoretical Approaches to the Theory of Voting," American Journal of Political Science, 24: 68-96.

[25] H. Moulin (1986) "Choosing from a Tournament," Social Choice and Welfare, 3: 271-291.

[26] D. Pearce (1984) "Rationalizable Strategic Behavior and the Problem of Perfection," Econometrica, 52: 1029-1050.

[27] C. Plott (1967) "A Notion of Equilibrium and Its Possibility Under Majority Rule," American Economic Review, 57: 787-806.

[28] W. Riker (1980) "Implications from the Disequilibrium of Majority Rule for the Study of Institutions," American Political Science Review, 74: $432-446$.

[29] A. Rubinstein (1979) "A Note on the Nowhere Denseness of Societies Having an Equilibrium Under Majority Rule," Econometrica, 47: 511514.

[30] W. Rudin (1966) Real and Complex Analysis, New York: McGraw-Hill.

[31] D. Saari (1997) "The Generic Existence of a Core for q-rules," Economic Theory, 9: 219-260.

[32] N. Schofield (1983) "Generic Instability of Majority Rule," Review of Economic Studies, 50: 695-705.

[33] M. Stinchcombe and H. White (1992) "Some Measurability Results for Extrema of Random Functions Over Random Sets," Review of Economic Studies, 59: 495-512. 


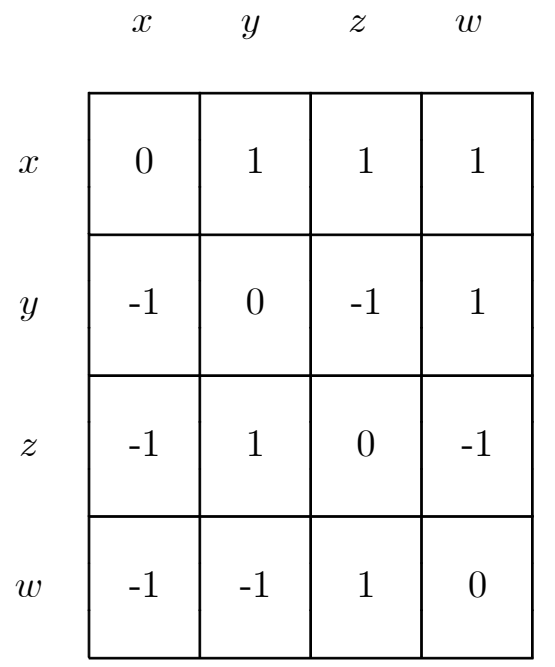

Figure 1: $x C z$ but $z$ is rationalizable.

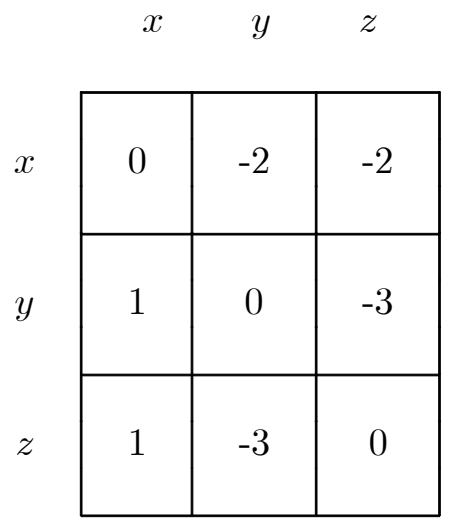

Figure 2: $x$ is $C$-maximal but not rationalizable. 


\begin{tabular}{|c|c|c|c|}
\hline$x$ & 0 & 1 & -1 \\
\hline$y$ & -1 & 0 & 0 \\
\hline$z$ & 1 & 0 & 0 \\
\hline
\end{tabular}

Figure 3: $y$ is played in equilibrium but $x M y$.

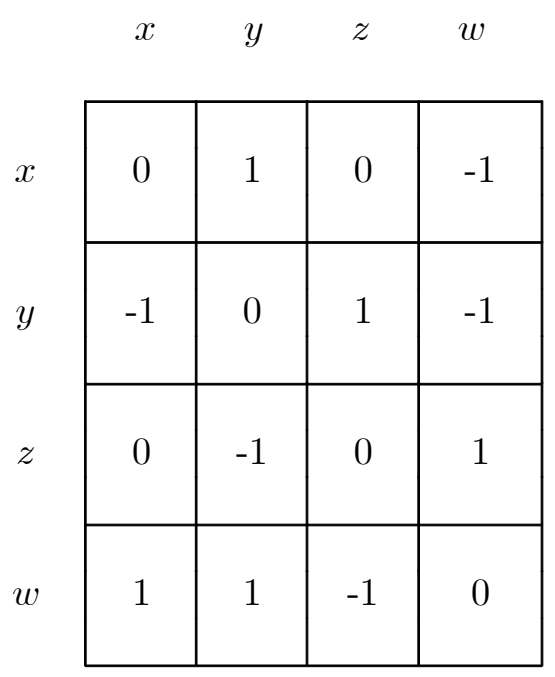

Figure 4: $y$ is played in equilibrium but $x G y$. 


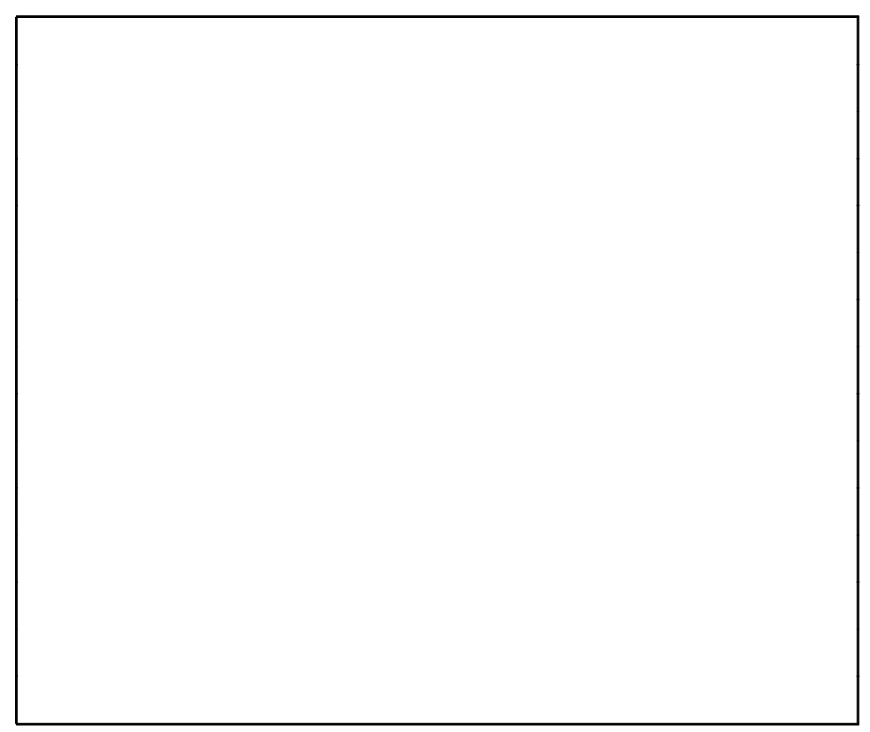

Figure 5: Nash equilibrium using covered strategies. 